DOI: $10.217672573-5365.100025$

\title{
Trastuzumab Combined Oxaliplatin and S-1 Therapy Demonstrated Pathological Complete Response of Synchronous Liver Metastasis of Gastric Cancer
}

\author{
Hiroshi Osawa ${ }^{1}$, Kenji Yamada ${ }^{2}$, Yoko Kuroda ${ }^{2}$ and Hisako Endo ${ }^{2}$ \\ ${ }^{1}$ Department of Oncology and Hematology, Edogawa Hospital, 2-24-18 Higashi-koiwa, Edogawa, Tokyo 133-0052, Japan \\ ${ }^{2}$ Department of Pathology, Edogawa Hospital, 2-24-18 Higashi-koiwa, Edogawa, Tokyo 133-0052, Japan
}

Corresponding author: Hiroshi Osawa, Department of Oncology and Hematology, Edogawa Hospital, 2-24-18 Higashi-koiwa, Edogawa, Tokyo 133-0052, Japan, Tel: 81336731221; Fax: 81336731229; E-mail: oosawa@edogawa.or.jp

Rec date: Jan 19, 2017; Acc date: Feb 02, 2017; Pub date: Feb 07, 2017

Citation: Osawa H, Yamada K, Kuroda Y, et al. Trastuzumab Combined Oxaliplatin and S-1 Therapy Demonstrated Pathological Complete Response of Synchronous Liver Metastasis of Gastric Cancer. Cell Mol Med. 2017, 3:1.

\section{Abstract}

The HER2 positive advanced or metastatic gastric cancer (GC) is recommended with Trastuzumab (Tmab) combined cisplatin (CDDP) and capecitabine (Cap). Otherwise, oxaliplatin (L-OHP) has been replaced in place of CDDP for GC. Furthermore, S-1 is a key oral anticancer drug used in the treatment of gastrointestinal cancer in Asia. A 59year-old man who received Tmab-SOX therapy for solitary synchronous liver metastasis of GC, because the operated intestinal type GC revealed HER2 score 3+ immunohistochemically. Tmab-SOX: (Tmab: $8 \mathrm{mg} / \mathrm{kg}$ for first cycle, 90minutes (mins) and $6 \mathrm{mg} / \mathrm{kg}$ for subsequent cycles on day 1,30 mins, L-OHP: $100 \mathrm{mg} / \mathrm{m}^{2}$, day 1,120 mins, S- $1: 80 \mathrm{mg} / \mathrm{m}^{2}$ twice daily, days 1 to 15 , orally, every 3 weeks). The solitary liver metastasis shrunk from $28 \mathrm{~mm}$ to $13 \mathrm{~mm}, 4$ cycles later. We performed right lobe hepatectomy which specimens' revealed pathological complete response. Tmab-SOX therapy proved remarkable response and might be an alternative chemotherapy plan against HER2 positive GC.

Keywords: S-1; Oxaliplatin; HER2 positive gastric cancer

\section{Introduction}

Gastric cancer (GC) still has a highly mortality rate and early stage resection has improved the outcome, which included HER2 positive GC ratio reported $21.1 \%$ in Japan [1]. S-1 is a key oral anticancer drug used in the treatment of an adjuvant and advanced or metastatic gastrointestinal cancer; the drug consists of a mixture of a prodrug of 5-fluorouracil (5-FU), namely tegafur, two modulators, viz. 5-chloro-2, 4dihydroxypyridine and potassium oxonate. S-1 exerts high antitumor effects and elicits fewer adverse events, especially fewer gastrointestinal adverse events than intravenous 5-FU. $\mathrm{S}-1$ has been replaced in place of 5FU in Asia [2]. Furthermore, oxaliplatin (L-OHP) has been replaced in place of CDDP for GC, reasons being senior patients, adverse event and hospitalization. Japanese investigators reported that L-OHP and S-1 (SOX) are active against advanced or metastatic GC with good response rate of $59 \%$ in a phase II [3] and $55.7 \%$ in a phase III [4]. Furthermore, HERBIS-1study [5] which consist of Trastuzumab (Tmab), cisplatin (CDDP) and S-1 (Tmab-SP) revealed good response rate and prolonged overall survivals against HER2 positive GC. Meanwhile, the SOX therapy has not been established against GC even HER2 positive GC. The HER2 positive metastatic $\mathrm{GC}$ is recommended with Tmab combined CDDP and capecitabine (Cap) (Tmab-XP) by ToGA study. However, L-OHP combined chemotherapy is realistic compared to the treatment of CDDP combined chemotherapy, are gone in present because senior patients, adverse event and hospitalization. And then, there are some reports of Tmab-LOHP and Cap (Tmab-XELOX) [6], there is no report of TmabSOX. Furthermore, we need alternative fluoropyrimidine chemotherapy plan such as adverse events. Here we have first reported pathological complete response in HER2 positive GC with liver metastasis of Tmab-SOX, which might be an alternative chemotherapy plan against HER2 positive GC.

\section{Case Report}

A 59-year-old man who was admitted to another hospital with the chief complaint of abdominal pain and syncope attack was diagnosed with having GC. The partial gastrectomy was performed on $1^{\text {st }}$ November 2015 at our hospital and still remained solitary synchronous liver metastasis. This study was carried out according to the regulation of local ethics a committee of our hospital and according to the declaration of Helsinki and provided written consent based on informed consent. He received Tmab combined L-OHP and S-1 (TmabSOX) therapy for solitary synchronous liver metastasis from 5 th January to $22^{\text {nd }}$ March 2016 as a neo-adjuvant chemotherapy. The first-line Tmab+SOX chemotherapy was initiated [tratuzumab (Tmab): $8 \mathrm{mg} / \mathrm{kg}$ for first cycle, 1.5 hours (h) intravenous infusion (iv) and $6 \mathrm{mg} / \mathrm{kg}$ for subsequent cycles on day 1, $30 \mathrm{~min}, \mathrm{v}$, oxaliplatin (L-OHP): $100 \mathrm{mg} / \mathrm{m}^{2}$ on day $1,2 \mathrm{~h}$ iv, S-1: $80 \mathrm{mg} / \mathrm{m}^{2}$ twice daily on days 1 to 15 , orally, every 3 weeks]. We did not recognize any adverse events during chemotherapy in outpatients' units. The tumor marker of 
CA72-4 (normal range: $0-10.0 \mathrm{U} / \mathrm{ml}$ ) decreased from 14.5 to 5.8 four cycles later. The solitary liver metastasis shrunk from $28 \mathrm{~mm}$ to $13 \mathrm{~mm}$ four cycles later (Figures 1a and 1b). We held a cancer board which discussed surgical resection with solitary liver metastasis. Therefore, we performed right hepatectomy on April $19^{\text {th }} 2016$.

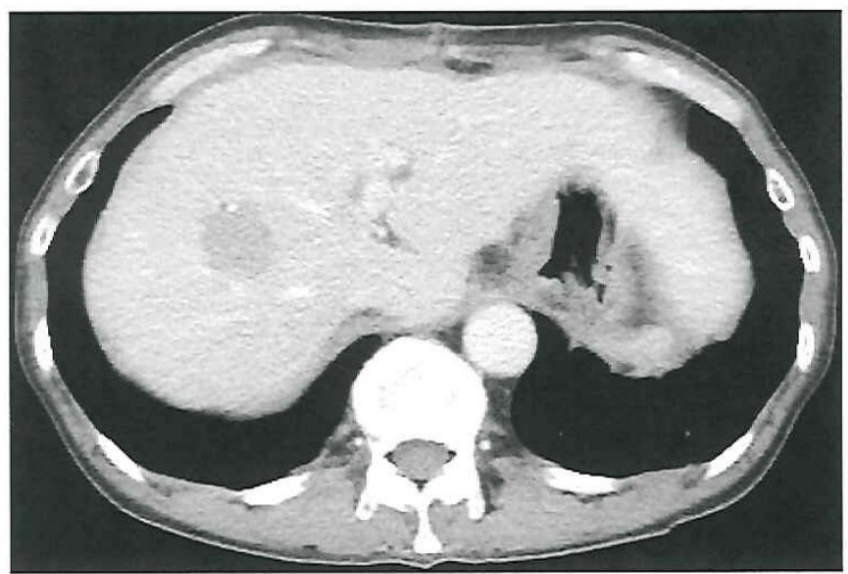

Fig.1a

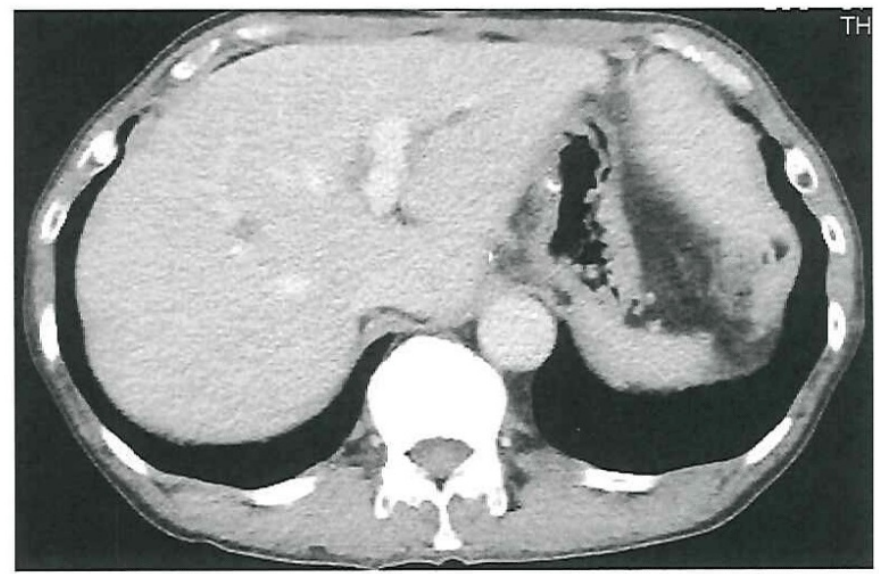

Fig.1b

Figure 1 (a) The computed tomography of the $28 \mathrm{~mm}$ of solitary liver metastasis on S8 area at first visit. (b) The computed tomography of the $13 \mathrm{~mm}$ of solitary liver metastasis on $\mathrm{S} 8$ area after 4 cycles chemotherapy.

\section{Pathological Findings}

Microscopically, the gastric cancer was predominantly composed of papillary adenocarcinoma (Lauren classification: intestinal type) with prominent vascular invasion (Figures $\mathbf{2 a}$ and $\mathbf{2 b}$ ). Immunohistochemically (IHC) study showed diffuse and strong HER2 positivity in almost entire tumor cells (HER2 IHC score 3+, Figure 2c). Fluorescence in situ hybridization (FISH) analysis revealed HER2 gene amplification with a
HER2:CEP17 ratio of 6.9. After 4 cycles of Tmab-SOX therapy, right hepatectomy was performed. Grossly, a yellow-white 12 $\mathrm{mm} \times 7 \mathrm{~mm}$ nodule was recognized in S8 of resected liver (Figure 3a). Histologically, the nodule was composed of the accumulation of foamy cells and fibrotic tissue with a loss of hepatocyte. Viable tumor cells were not observed (Figures $\mathbf{3 b}$ and $3 c$ ). These findings were compatible with the complete eradication of metastatic GC by Tmab-SOX therapy.

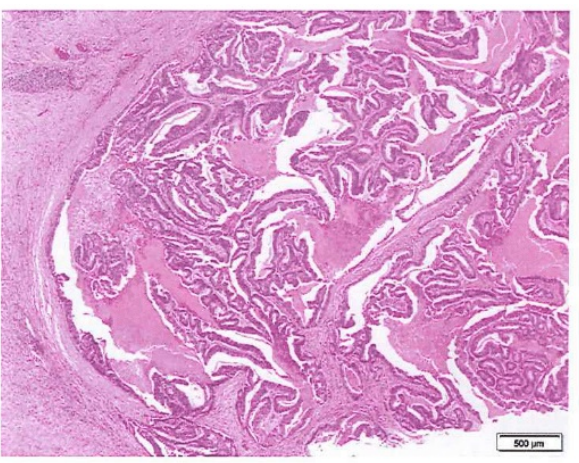

Fig.2a

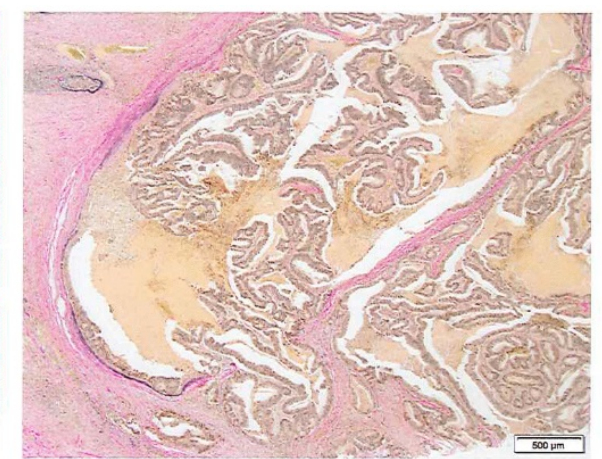

Fig.2b

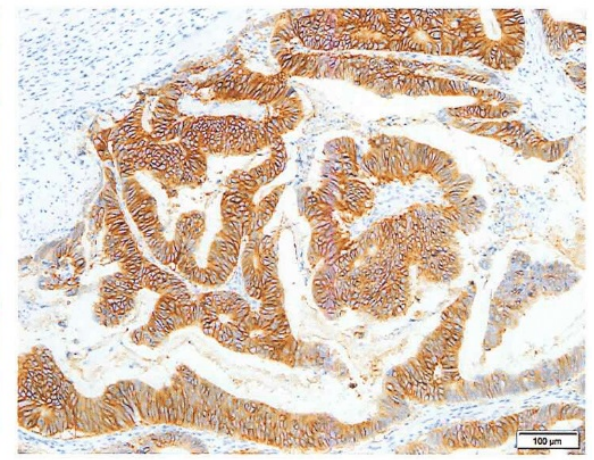

Fig.2c

Figure 2 Microscopically, the gastric cancer was predominantly composed of papillary adenocarcinoma with prominent vascular invasion (a): H\&E, x20, (b): Elastica van Gieson, x20). (c) Immunohistochemical study showed diffuse and strong HER2 positivity in almost entire tumor cells (HER2 IHC score $3+, \mathrm{x} 100$ ). 


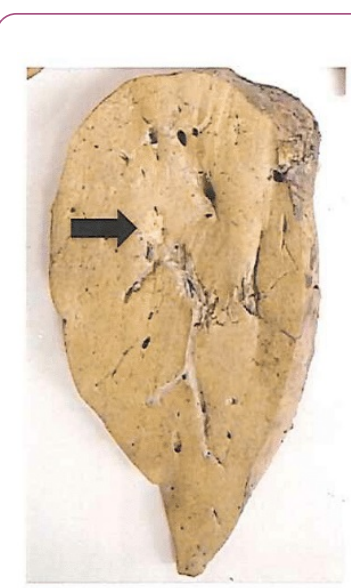

Fig.3a

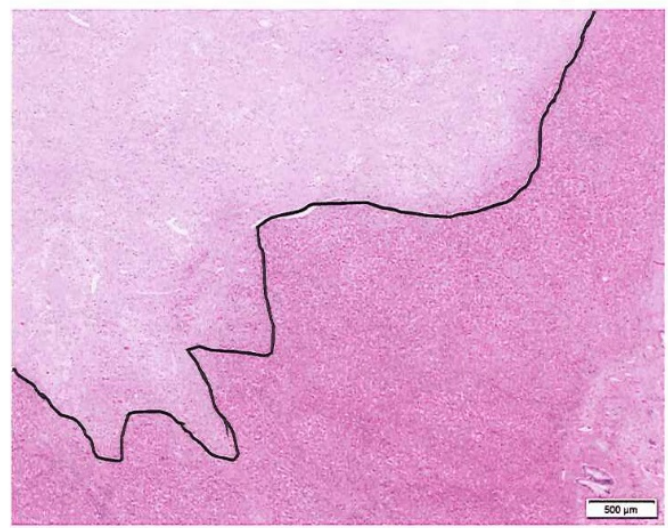

Fig.3b

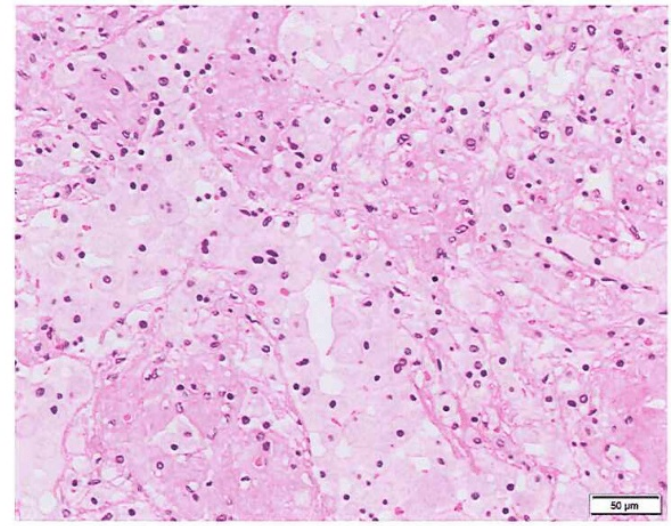

Fig.3c

Figure 3 (a) Grossly, a yellow-white $12 \mathrm{~mm} \times 7 \mathrm{~mm}$ nodule was recognized in S8 of resected liver. (b and c) Histologically, the nodule was composed of the accumulation of foamy cells and fibrotic tissue with a loss of hepatocyte. Viable tumor cells were not observed. (H\&E, b: x20, c: x200).

\section{Discussion}

A patient of $\mathrm{GC}$ with liver metastasis is poor prognosis, whose 5-year survival rate went from 10 to $40 \%$ after liver metastasis resection [7]. Otherwise, these cases have a selection biases such as small numbers of patients, small numbers of metastasis and retrospective studies. Regardless, the liver metastasis with colorectal cancer has been established, the hepatectomy with GC is controversial due to is biologic aggressiveness of the disease such as peritoneal dissemination, multiple liver metastases in both lobes, lymphnodes metastases. However, Oki et al. reported that solitary metastasis was an independent prognostic factor in multicenter cohort analysis [8]. We consider that neoadjuvant chemotherapy is one of the effective methods for solitary liver metastasis and HER2 positive GC in our case because ToGA study revealed that Tmab group showed a $26 \%$ reduced mortality rate, 2.7 months increase in overall survival time and a $12 \%$ increase in objective response rates. Although the heterogeneity of HER2 protein expression and HER2 gene amplification within a same tumor is a common finding in GC [9], the present case showed broad and uniform overexpression of HER2 protein and greater than 6-fold amplification of HER2 gene. These findings may explain the pathological complete response in the present case. We had selected Tmab-SOX which reasons such as 1) S-1 is a key oral anticancer drug used in the treatment of gastrointestinal cancer in Asia, 2) SOX reported good response rate in phase II/III trials compared to CDDP+S-1, 3) Li et al. reported that Tmab-SOX was remarkable response of synchronous liver metastasis of poorly differentiated HER2 positive gastric cancer (Lauren classification: diffuse type) [10], 4) We previously treated that the doublet combination of SOX is active against advanced or metastatic colorectal cancer. 5) If a patient had some severe adverse events due to L-OHP, S-1 alone therapy has strong evidence against GC. The patient was treated with Tmab-SOX for four cycles and demonstrated 54\% shrinkage, which result revealed a partial response by RECIST.
We performed right hepatectomy for the radical cure of GC. Surprisingly, pathological examination revealed complete replacement of the remaining $12 \mathrm{~mm}$ nodule with foamy cells and fibrous tissue without viable tumor cells. In our case, we treated the Tmab-SOX regimen in a liver metastatic GC patient which resulted in an improved quality of life without recurrence. Actually, there are several reports of Tmab-XELOX regimen against advanced GC patients. Furthermore, there is the ongoing phase II trial of Tmab-SOX therapy for HER2 positive advanced GC in Japan (UMIN000021209). If this study is to succeed, neo-adjuvant chemotherapy with Tmab-SOX, for HER2-positive GC, should be further investigated. Actually, many investigators reported that Tmab with XP and SP revealed good response rate and prolonged overall survivals against HER2 positive GC. Otherwise, these reports had not recognized pathological CR [11,12]. Tmab with triplet chemotherapy (docetaxel, cisplatin and S-1:DCS) demonstrated good pathological response and survival rate. The other hand, Tmab with SP, XP and DCS are inconvenience to treat which reasons are senior patients, adverse event and hospitalization. However, Tmab with XELOX and SOX are convenient to treat for any GC patient even outpatients' units. Furthermore, we need alternative fluoroprimidine chemotherapy plan such as adverse events which are Hand-Foot-syndrome with Cap and gastrointestinal toxicities with S-1. Finally, this is the first reported case of a solitary liver metastasis of HER2 positive GC completely eradicated by Tmab-SOX therapy. We did not recognize any adverse events during chemotherapy in outpatients' units. Tmab-SOX therapy proved remarkable response and might be an alternative chemotherapy plan against HER2 positive GC.

\section{Conflict of Interest}

All authors of this manuscript have no conflict of interest statement. 


\section{Ethical Standards}

Human rights statement and informed consent.

\section{References}

1. Matsusaka S, Nashimoto A, Nishikawa K, Miki A, Miwa $H$, et al. (2016) Clinicopathological factors associated with HER2 status in gastric cancer results from a prospective multicenter observational cohort study in a Japanese population (JFMC44-1101). Gastric cancer 19: 839-851.

2. Koizumi W, Takiuchi H, Yamada Y, Boku N, Fuse N, et al. (2010) Phase II study of oxaliplatin plus S-1 as first-line treatment for advanced gastric cancer (G-SOX study). Ann Oncol 21: 1001-1005.

3. Yamada Y, Higuchi K, Nishikawa K, Gotoh M, Fuse N, et al. (2015) Phase III study comparing oxaliplatin plus S-1 with cisplatin plus S-1 in chemotherapy-naïve patients with advanced gastric cancer. Ann Oncol. 26: 141-148.

4. Kurokawa $\mathrm{Y}$, Sugimoto $\mathrm{N}$, Miwa H, Tsuda M, Nishina S, et al. (2014) Phase II study of trastuzumab in combination with S-1 plus cisplatin in HER2-positive gastric cancer (HERBIS-1). $\mathrm{Br} J$ Cancer. 110: 1163-1168

5. Ryu MH, Yoo C, Kim JG, Ryoo BY, Park YS, et al. (2015) Multicenter phase II study of trastuzumab in combination with capecitabine and oxaliplatin for advanced gastric cancer 51 : 482-488.

6. Takemura N, Saiura A, Koga R, Arita J, Yoshioka R, et al. (2012) Long-term outcomes after surgical resection for gastric cancer liver metastasis and analysis of 64 macroscopically complete resections. Langenbecks Arch Surg. 397: 951-957.

7. Oki E, Tokunaga S, Emi Y, Kusumoto T, Yamamoto M (2016) Surgical treatment of liver metastasis of gastric cancer: a retrospective multicenter cohort study (KSCC1302). Gastric Cancer. 19: 968-976.

8. Kim MA, Lee HJ, Yang HK, Bang YJ, Kim WH (2011) Heterogeneous amplification of ERBB2 in primary lesions is responsible for the discordant ERBB2 status of primary and metastatic lesions in gastric carcinoma. Histopathology. 59: 822-831.

9. Li ZY, Shan F, Zhang ZD, Bu ZD, Wu AW (2014) Preoperative chemotherapy with a trastumab-containing regimen for a patient with gastric cancer and hepatic metastases. GMR 13: 10952-10957.

10. Choda Y, Ninomiya M, Kanazawa T, Sato D, Tokumoto N (2014) Gastric cancer with liver metastasis and peritoneal dissemination with conversion surgery to achieve Ro resection after capecitabine, cisplatin, and trastuzumab chemotherapy- A case report. Gan To Kagaku Ryoho 41(11): 1421-1424.

11. Kim Y, Hosoda Y, Nishio M, Okano M, Kawada J, et al. (2015) Hepatic resection of multiple liver metastases from gastric cancer after molecular targeted chemotherapy. Gan To Kagaku Ryoho 42(12): 1635-1637.

12. Mitsui $Y$, Sato $Y$, Miyamoto $H$, Fujino $Y$, Takaoka T (2015) Trastuzumab in combination with docetaxel/cisplatin/S-1 (DCS) for patients with HER2-positive metastatic gastric cancer: feasibility and preliminary efficacy. Cancer Chemother Pharmacol 76: 375-382. 